\title{
Los bañados de desborde fluvial: Una mirada desde la geohidrología
}

\author{
Leandro Rodrigues Capítulo', Eduardo Kruse ${ }^{1}$ y Nora Gómez ${ }^{2}$ \\ ${ }^{1}$ Consejo Nacional de Investigaciones Científicas y Técnicas (CONICET). Centro de Estudios Integrales \\ de la Dinámica Exógena (CEIDE), Facultad de Ciencias Naturales y Museo, Universidad Nacional \\ de La Plata (UNLP), La Plata, Argentina. \\ 2Instituto de Limnología “Dr. Raúl A. Ringuelet” (CONICET - UNLP), La Plata, Argentina
}

E-mail: leandrorodriguescapitulo@gmail.com

\begin{abstract}
RESUMEN. Los bañados de desborde fluvial (BDF) abarcan tramos (cientos de metros a kilómetros) de los sistemas fluviales pampeanos, con características y funciones particulares. Estos humedales se originan por un afloramiento del nivel freático y una disminución local del gradiente hidráulico, lo que da lugar a un ensanchamiento del curso de agua principal y la aparición de una red de microdrenaje que acompaña a este último. Estas condiciones favorecen el desarrollo de humedales asociados al curso principal, con un importante desarrollo de la vegetación característica de suelos muy húmedos. El objetivo de este trabajo fue el de analizar las características hidrológicas y la calidad del agua de los BDF en zonas urbanizadas con actividad agrícola (localizados en los arroyos Carnaval y Del Gato) y en zonas rurales con ganadería extensiva (ubicados en los arroyos Cajaravillas y Chubichaminí), del NE de la provincia de Buenos Aires, con el fin de reconocer cómo los diferentes usos del suelo modifican las condiciones de conservación de los humedales. Los análisis geohidrológicos se realizaron a escala regional (cuenca), semi-detalle (subcuenca) y detallada (BDF). Los resultados mostraron que la sobreexplotación de las aguas subterráneas afecta a la permanencia del agua en los humedales, debido a la desconexión entre el nivel freático y el nivel basal de los BDF situados en las zonas urbanizadas con actividad agrícola. En estos últimos se ha observado también que el aporte de nutrientes contribuye significativamente al deterioro de la calidad del agua. La conclusión es que un diagnóstico ambiental de estos BDF requiere un enfoque regional de los procesos hidrológicos para adoptar acciones adecuadas para su conservación.
\end{abstract}

Palabras clave: Agua subterránea, agricultura, calidad del agua, hidrología, urbanización.

ABSTRACT. The riverine wetlands (RWs) cover stretches (ranging from hundreds of meters to kilometers) of the Pampean river systems, with particular characteristics and functions. These wetlands are the result of an outcropping of the phreatic level and a local decrease in the hydraulic gradient, which gives rise to a widening of the main watercourse together with the appearance of a micro-drainage network. These conditions favor the development of wetlands associated with the main course, with a significant development of the typical vegetation of very wet soils. The aim of this study was to analyze the hydrological characteristics and water quality of the RWs located in urbanized areas with agricultural activity (in Carnaval and Del Gato streams) and in rural areas with extensive cattle farming (in Cajaravillas and Chubichamini streams), in order to recognize how different land uses modify the conservation conditions of the wetlands. The geohydrological analyses were carried out on a regional (basin), semi-detailed (sub-basin) and detailed (RWs) scale. The results showed that the overexploitation of groundwater affects the permanence of water in the wetlands, due to the disconnection between the phreatic level and the basal level of the RW 
located in the urbanized area with agricultural activity. In these latter, the supply of nutrients also influences the deterioration of water quality. The conclusion is that an environmental diagnosis of these RWs requires a regional approach to hydrological processes in order to adopt adequate actions for their conservation.

Keywords: Hydrology, groundwater, water quality, agriculture, urbanization.

\section{INTRODUCCIÓN}

Los sistemas fluviales a lo largo de su recorrido hacia el mar interactúan con el valle aluvial que lo circunda, adquiriendo características específicas de acuerdo con la geología y la pendiente, que a su vez son moduladas por la variabilidad temporal. En las grandes llanuras, como la pampeana, el rasgo distintivo que condiciona la hidrología es la escasa pendiente del terreno. Esto ha llevado a reconocer a este tipo de ambientes como sistemas hidrológicos no típicos (SHNT) (Fertonani \& Prendes, 1983), en los cuales no es posible identificar una red de drenaje integrada, un escurrimiento superficial definido por cursos de agua discretos o procesos erosivos fluviales. Como consecuencia de ello los métodos de cuantificación, medición y pronósticos presentan adaptaciones particulares que los diferencian. En este contexto, las aguas superficiales y subterráneas conforman una unidad, en donde los movimientos verticales del agua prevalecen sobre los horizontales, estando expuestos a las variaciones climáticas que conducen a excesos y déficit de agua (Kruse \& Zimmermann, 2002). El clima húmedo de la región suele determinar la inundación temporaria del territorio conduciendo al anegamiento del suelo el cual se manifiesta a partir del desarrollo de cuerpos de agua someros, que están vinculados directa o indirectamente a los cursos de agua.

En los sistemas fluviales pampeanos del NE de la Provincia de Buenos Aires es posible identificar tramos que pueden abarcar desde un centenar de metros hasta kilómetros, denominados como bañados de desborde fluvial (BDF), con características y funciones particulares. En condiciones naturales este tipo de ambientes se generan a partir del afloramiento del nivel freático y por una disminución local del gradiente hídrico (diferencia de alturas hidráulicas de un curso de agua), lo cual se traduce en un ensanchamiento del curso de agua principal y la aparición de una red de microdrenaje, que acompaña a este último. Esta red permanece inactiva durante algunos períodos en ausencia de precipitaciones, reactivándose durante etapas lluviosas cuando la intensidad de estas supera la capacidad de infiltración del terreno. Esta condición favorece la generación de humedales asociados al curso principal que presentan un importante desarrollo de vegetación característica de suelos muy húmedos manifestados a partir de rasgos de hidromorfismo. Estos "ecotramos", de acotada expresión en el terreno, cuyo anegamiento en superficie se expande y contrae periódicamente, tienen una estructura biótica que es producto de las interacciones bidireccionales entre el curso principal y la planicie de inundación, que a su vez dependen estrechamente de la descarga del agua subterránea.

El avance de la urbanización y las prácticas agrícolas han ido destruyendo o alterando este tipo de ambientes en la llanura y con ello modificando los beneficios que prestan como por ejemplo a los procesos de depuración (Cochero et al., 2020; Gasparini et al., 2019; Gómez et al., 2016; Laterra et al., 2018) como hábitaty refugio para la biota (Gómez et al., 2016) y la regulación de crecidas (Kruse y Laurencena, 2005), entre otros. Teniendo en cuenta estas consideraciones en este trabajo se propone la caracterización geohidrológica de casos de estudio contrastantes de BDF expuestos a diferentes usos del suelo. Para lograr estos objetivos se seleccionaron dos BDF localizados en una zona rural y otros dos influenciados por la agricultura y la urbanización, ubicados en el noreste de la provincia de Buenos Aires. La finalidad de este estudio es reconocer como las 
intervenciones humanas, en las cuencas donde se desarrollan estos humedales, modifican sus características naturales.

\section{Materiales y métodos Área de estudio}

El área de estudio se encuentra localizada en la Ilanura pampeana, en el NE de la Provincia de Buenos Aires. El clima es húmedo a subhúmedo, con una precipitación media anual de 1060 mm/ año y una evapotranspiración de 783 mm/año. La precipitación constituye el principal ingreso de agua a considerar en el balance hidrológico de esta zona (Laurencena et al., 2010).

Se trata de un ámbito llano de escasa pendiente, en donde el acuífero freático se halla a poca profundidad, entre 1 y 3 m b.n.t., (metros bajo el nivel del terreno) y presenta un escurrimiento local hacia los cursos de agua y regionalmente hacia el estuario Río de la Plata (Figura 1). Los gradientes hídricos medios se encuentran en un rango de 0,6 a los 0,9 m/km.

Los BDF analizados en este trabajo están vinculados a tramos de los arroyos cuya extensión es de aproximadamente 1000 m de longitud y en donde el gradiente hídrico disminuye progresivamente dando lugar a la ampliación del cauce. Esta condición se pone de manifiesto a partir de la aparición de humedales en forma alargada y cuyo ancho es variable.

Los BDF seleccionados se clasificaron según los usos de suelo en dos grupos, uno de ellos situados en la zona periurbana (BDF Carnaval y BDF del Gato) y el otro en zonas rurales (BDF Cajaravillas y BDF Chubichaminí).

El grupo situado en la zona periurbana está asociado a las cuencas de los arroyos Carnaval y del Gato, ubicándose en los suburbios de la ciudad de La Plata, distantes a 7 km y 3 km respectivamente de sus cabeceras (Figura 1).

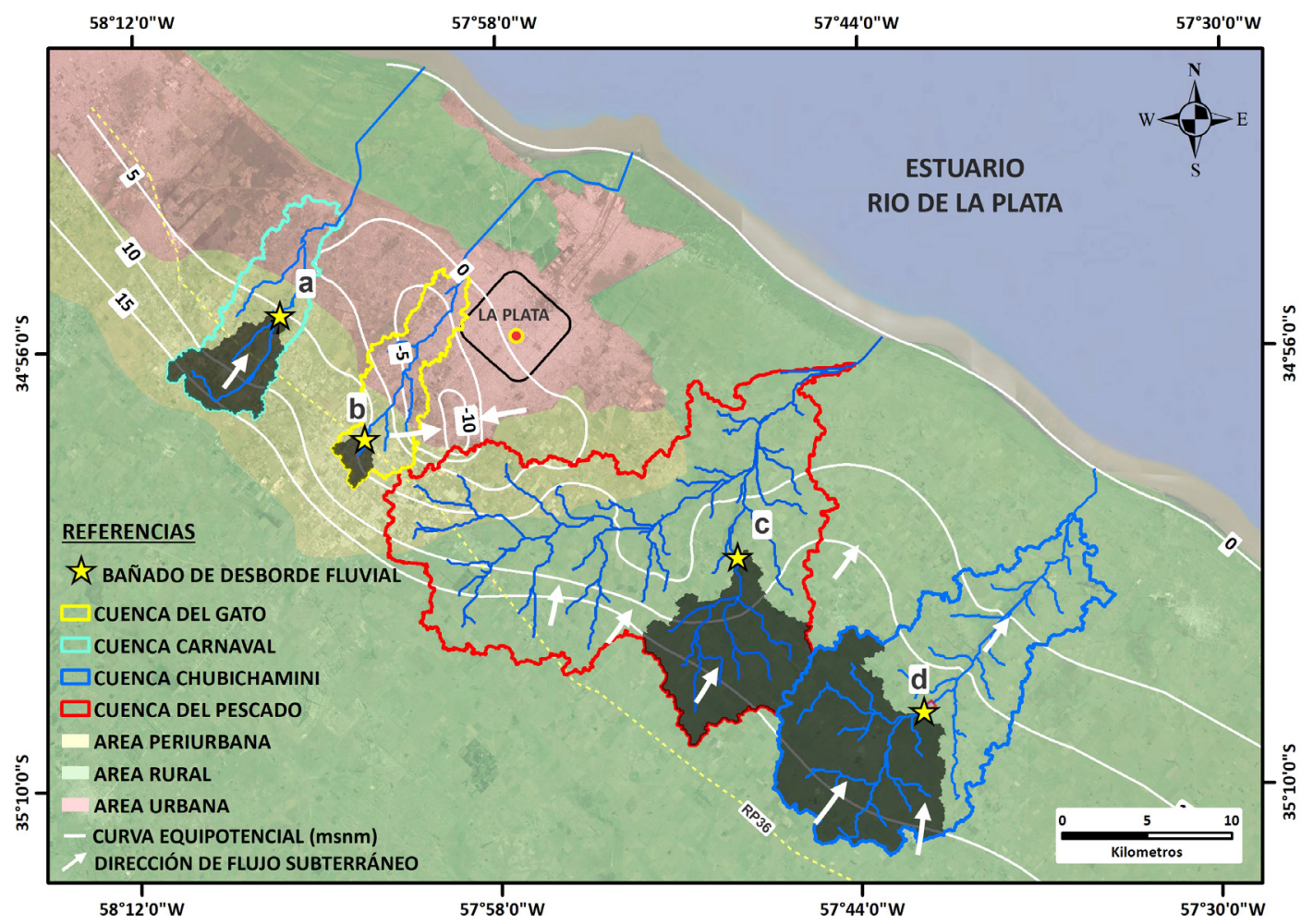

Figura 1. Área de estudio. Ubicación de las cuencas, subcuencas y bañados de desborde fluvial. a. BDF Carnaval, b. BDF Del Gato, c. BDF Cajaravillas y d. BDF Chubichaminí.

Figure 1. Study area. Location of studied watersheds, sub-basins and riverine wetlands. a. RW Carnaval, b. RW Del Gato, c. RW Cajaravillas and d. RW Chubichaminí. 
El primero se encuentra rodeado por cultivos de soja, el segundo se encuentra influenciado por la actividad hortícola. Aguas abajo, ambos arroyos atraviesan zonas pobladas y sus cauces han sido fuertemente intervenidos por la acción del hombre (canalizaciones, entubamientos, etc) condicionando su vinculación con el estuario del Río de la Plata, donde desaguan.

El otro grupo ubicado en zonas rurales se corresponden con las cabeceras de las cuencas de los arroyos Chubichaminí y Cajaravillas, respectivamente (Figura 1). Se han estimado a partir de aforos puntuales, caudales en ambos arroyos de $0,4 \mathrm{~m}^{3} / \mathrm{s}$. Ambos cursos de agua drenan hacia el estuario del Río de la Plata atravesando zonas rurales con escasa intervención antrópica en su escurrimiento superficial. El uso del suelo es rural con ganadería extensiva ( 0,7 vacas /ha).

El agua subterránea de ambas cuencas presenta características bicarbonatadas sódicas, evolucionando a clorurada sódica hacia la zona de descarga. El agua superficial también posee agua de características bicarbonatadas sódicas que pasan gradualmente a cloruradas sódicas, que en algunas oportunidades pueden presentar alta turbiedad producto del transporte en suspensión de materia orgánica (Bazán et al., 2011).

\section{Muestreos y análisis de la información}

En la metodología adoptada se planteó un análisis en sucesivas aproximaciones, partiendo de una caracterización regional para progresivamente avanzar hacia un análisis detallado de los BDF seleccionados.

Es así que se tuvieron en cuenta distintas escalas de análisis, según los siguientes niveles:

1. Cuenca hidrográfica (escala regional), vinculada a las características generales de la unidad hidrológica.

2. Subcuenca de aporte al BDF (escala de semidetalle), integrando las variables hidrológicas que se manifiestan entre las cabeceras y los sitios de estudio.

3. BDF (escala de detalle), abordando las particularidades de los bañados propiamente dichos.

La caracterización regional y de semidetalle se realizó a través de imágenes de satélite de Google Earth. Su delimitación se llevó a cabo utilizando un DEM (Modelo de Elevación Digital) de una imagen capturada por el satélite ALOS, equipado con un sensor de radar "PALSAR" (Radar de Apertura Sintética de Banda L Tipo Phased Array) con una resolución espacial de $12,5 \mathrm{~m}$.

Para el análisis a escala de detalle se realizaron vuelos aerofotogramétricos mediante el empleo de un dron multirotor Phantom IV Pro equipado con un sensor RGB de alta definición, sensor infrarrojo para detección de obstáculos, sistema estabilizador automático en vuelo, altura máxima de $500 \mathrm{~m}$ snt (metros sobre el nivel del terreno) y una velocidad límite de 15 $\mathrm{m} / \mathrm{s}$. De este modo, la configuración del vuelo contempló la mejor relación de solapamiento de fotogramas, altura de vuelo, velocidad de vuelo, grado de detalle del pixel, entre otros.

La elección de la grilla de vuelo en los casos estudiados incluyo como criterio las bajas pendientes del terreno (menores al 1\%) que caracterizan a la llanura pampeana. Con relación al solapamiento y con el objeto de garantizar la fiabilidad tanto de los ortomosaicos como también del modelo digital de elevación, se seleccionó el distanciamiento óptimo entre transectas de vuelo permitiendo un solapamiento mayor al $70 \%$. La velocidad de vuelo se fijó con la finalidad de permitir el relevamiento de toda el área mediante el empleo de una única batería.

Para un relevamiento del orden de $30 \mathrm{~h}$ se emplearon grillas de $1000 \mathrm{~m}$ de longitud por $300 \mathrm{~m}$ de ancho. La tarea se realizó a una altura de vuelo de $120 \mathrm{~m}$ snt, en menos de 15 minutos, obteniéndose aproximadamente 300 fotogramas conseguidos a partir de 6 o 7 transectas.

La verificación de los valores planialtimétricos obtenidos con el dron requirieron el acotamiento de puntos de control situados en sectores estratégicos. Los puntos acotados, con una precisión de centímetros, sirvieron como red de apoyo para validar los datos obtenidos por el dron durante el post-proceso en gabinete.

Para el procesamiento de los fotogramas se emplearon softwares de fotogrametría a partir de los cuales se confeccionó un modelo digital de elevación (DEM) y un ortomosaico 
georreferenciado y ortorectificado que integra todas las tomas realizadas resultando en una imagen de detalle con una resolución espacial del orden $3 \mathrm{~cm} /$ pixel.

Para la transformación del DEM a MDT (Modelo digital del terreno) en primer lugar se realizó la clasificación de los puntos del terreno. Este proceso permite discriminar aquellos pixeles asociados a la vegetación de aquellos que resultan del terreno propiamente dicho. Posteriormente se creó una malla en donde los puntos asociados a la vegetación fueron excluidos permitiendo obtener mapas de curvas topográficas acordes al terreno estudio.

Para la integración de la información generada se emplea un sistema de información geográfica, a partir del cual fue posible la creación de diferentes capas raster y vectoriales consistentes en mapas de dirección y acumulación de flujo; delimitación de cuencas e identificación de la red drenaje principal y secundaria cuyos productos permitieron el análisis con diferentes escalas de detalle.

Adicionalmente, se realizaron sondeos manuales cuya profundidad final alcanzó los $8 \mathrm{~m}$ b.n.t. Para llevar a cabo dicha tarea se empleó un barreno tipo "cola de pescado" a partir del cual se identificó la posición del nivel freático y además se obtuvieron muestras de cutting de perforación. Se elaboraron mapas isofreáticos para determinar la relación entre los BDF y el agua subterránea. En aquellos sectores ubicados en áreas con niveles freáticos someros, se ejecutaron perforaciones de monitoreo en donde se instalaron sensores de nivel automáticos (Solinst, Modelo 3001 - LTC Levelogger Edge) los cuales posibilitaron la obtención de registros continuos de los niveles hidráulicos. Adicionalmente se extrajeron muestras de agua subterránea $(500 \mathrm{ml})$ para la caracterización hidroquímica (cationes y aniones) que fueron analizados según la metodología propuesta por Clesceri et al. (1998). En aquellos que no están relacionados con el agua subterránea, se utilizaron pozos de extracción de agua cercanos a los BDF.

Para la caracterización de los usos del suelo se emplearon los criterios de Laurencena et al. (2010). Los datos de temperatura y lluvia se registraron a partir de la información de una estación meteorológica (Davis Vantage Pro2), instalada en el área de estudio.

Con la finalidad de caracterizar la calidad del agua superficial se realizaron muestreos en otoño (marzo de 2017), invierno (junio de 2017), primavera (noviembre de 2017) y verano (febrero de 2018) en las cuatro BDF. En cada muestreo se midieron in situ $\mathrm{pH}$, conductividad, turbidez, porcentaje de saturación de oxígeno disuelto y la temperatura. También se extrajeron muestras de agua superficial por triplicado para la determinación de fósforo reactivo soluble, nitratos, nitritos, amonio, DBO5 y DQO, que fueron refrigeradas y transportadas hasta el arribo al laboratorio. Los nutrientes se analizaron según Mackereth et al. (1978) y las demandas de oxígeno según Clesceri et al. (1998).

Las diferencias de las variables físico-químicas se analizaron mediante un ANOVA unidireccional y la prueba de Tukey a posteriori $(p<0,05)$, cuando se cumplieron los supuestos de normalidad y homocedasticidad. En los casos en que los datos no cumplían con estos supuestos, se realizó ANOVA unidireccional en los rangos (KruskalWallis), seguido a posteriori por la prueba de Dunn $(p<0,05)$

\section{RESULTADOS \\ Cuencas hidrográficas}

El sistema hidrológico regional se vincula a cuencas cuyas cabeceras coinciden con la traza de la Ruta 36, presentando una forma alargada en sentido SO-NE y drenando sus aguas hacia el estuario del Río de la Plata (Figura 1). Las cuencas hidrográficas en las cuales se incluyen los cuatro casos de estudio seleccionados se caracterizan por los parámetros morfométricos que se indican en las Tablas 1 y 2.

El flujo subterráneo se produce regionalmente hacia el estuario, aunque en el sector $\mathrm{S}$ del casco urbano de La Plata se identifica un cono de depresión el cual se vincula con la extracción intensiva de aguas subterráneas cuyo ápice se centra en la localidad de Los Hornos y alcanza una profundidad mayor a los -10 msnm (Figura 1).

La característica principal de las cuencas del 
Tabla 1. Parámetros de las cuencas de los humedales estudiados.

Table 1. Parameters of the basins of the studied wetlands.

\begin{tabular}{lcccc}
\hline & Carnaval & Del Gato & Pescado & Chubichaminí \\
\hline Área $\left(\mathrm{km}^{2}\right)$ & 54,6 & 42,7 & 337,0 & 190,6 \\
Perímetro $\left(\mathrm{km}^{2}\right)$ & 50,3 & 48,1 & 138,8 & 97,5 \\
Longitud del curso principal & 16,2 & 13,1 & 32,7 & 25,3 \\
Densidad de drenaje & 0,57 & 0,56 & 0,61 & 0,56 \\
Kc (Coeficiente de forma) & 0,21 & 0,25 & 0,32 & 0,30 \\
Diferencia de altura (m) & 10,5 & 11,6 & 13,6 & 9,4 \\
Pendiente promedio (m) & 0,06 & 0,09 & 0,04 & 0,04 \\
Tiempo de concentración (h) & 10,8 & 8,6 & 20,4 & 17,1 \\
\hline
\end{tabular}

primer grupo de humedales (Carnaval y Del Gato) se asocia a una red de drenaje que ha sido fuertemente modificada a partir de rectificaciones, canalizaciones, dragados y entubados. Las diferencias de altura entre la zona de cabecera y la desembocadura alcanzan los 11 metros y se manifiestan en el terreno a partir de pendientes relativamente bajas en un rango entre 0,6 y 0,9 m/km en las cuencas de los arroyos Carnaval y Del Gato respectivamente generando tiempos de concentración que se encuentran entre 10 y 8 horas (Tabla 1).

Las cuencas del segundo grupo (Cajaravillas y Chubichaminí), presentan superficies significativamente mayores (Tabla 1), pendientes ligeramente menores que las descriptas anteriormente con valores que rondan los 0,4 $\mathrm{m} / \mathrm{km}$ y que conservan su morfología natural en toda su extensión. Con diferencias de alturas de entre los 13 y $9 \mathrm{~m}$, los tiempos de concentración alcanzan las 20 y 17 horas en las cuencas de los arroyos El Pescado y Chubichaminí, respectivamente.

\section{Subcuencas de aportes a los BDF}

Las características de las subcuencas que alimentan a los humedales Carnaval - Del Gato presentan superficies relativamente pequeñas alcanzando 24,2 y $4,3 \mathrm{~km}^{2}$ respectivamente (Tabla 2). Esta condición permite inferir bajos caudales, así como cortos tiempos de respuesta ante eventos de precipitación. Como consecuencia de la extracción intensiva de aguas subterráneas para el abastecimiento de la población y la producción frutihortícola se produce la desaparición de cursos de carácter permanente. Cabe destacar que, adicionalmente, una parte del caudal de estos cursos de agua tiene su origen por el aporte de aguas residuales y de los excedentes del riego. En el caso del arroyo Del Gato, tanto la canalización e impermeabilización del lecho, así como el emplazamiento de las industrias en los sectores aledaños al curso principal, ha generado la permanencia de un caudal base que se asocia principalmente al vertido de aguas residuales. Esto permitiría explicar la existencia de un flujo de agua semipermanente, incluso en períodos sin precipitaciones.

Las subcuencas de aporte a los BDF Cajaravillas y Chubichaminí son de una mayor extensión y el caudal base es aportado principalmente por la descarga del acuífero freático, que se encuentra a escasos centímetros por debajo del terreno. Los cursos se caracterizan por su carácter permanente, aún durante períodos de sequía, con un caudal base constante y un mayor desarrollo en amplitud de los BDF identificados (Tabla 2, Figura 2).

\section{Bañados de desborde fluvial}

En la Tabla 3 se muestran las principales particularidades de cada uno de los humedales.

Tabla 2. Parámetros de las subcuencas de los humedales estudiados.

Table 2. Parameters of the sub-basins of the studied wetlands.

\begin{tabular}{lcccc}
\hline & Carnaval & Del Gato & Cajaravillas & Chubichaminí \\
\hline Área de aporte $\left(\mathrm{km}^{2}\right)$ & 24,26 & 4,30 & 60,84 & 88,81 \\
Diferencia de altura & 6,1 & 5,5 & 8,3 & 6,6 \\
\hline
\end{tabular}




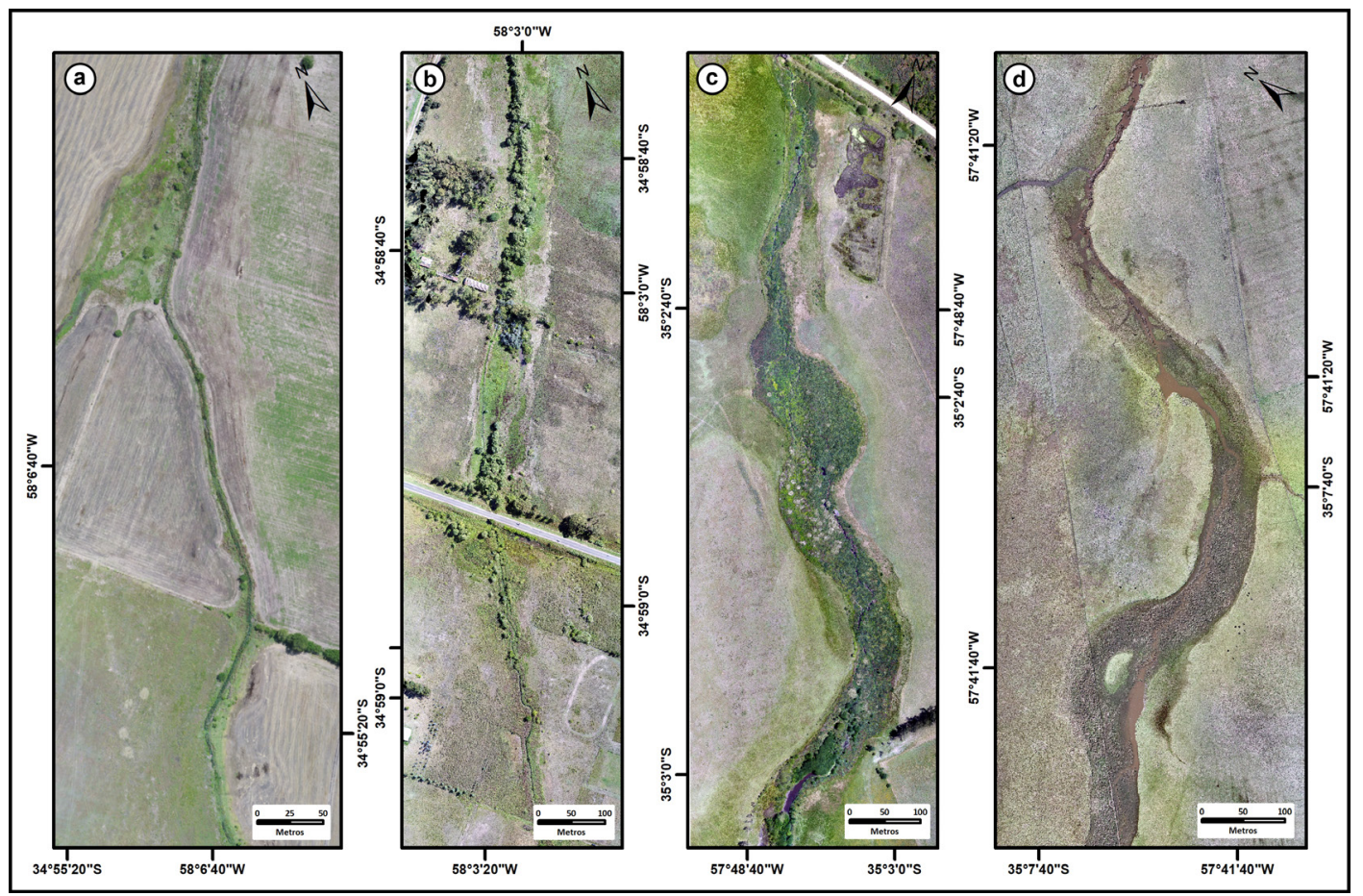

Figura 2. Relevamientos fotogramétricos realizados sobre los humedales ubicados sobre los arroyos a. Carnaval, b. Del Gato, c. Cajaravillas y d. Chubichaminí.

Figure 2. Photogrammetric surveys carried out on the riverine wetlands located on the a. Carnaval, b. Del Gato, c. Cajaravillas and d. Chubichaminí streams.

La manifestación de los BDF es localizada en relación con ligeras variaciones de la pendiente del curso de agua. Esta condición se traduce en una disminución de la energía y velocidad de flujo y por tanto un ensanchamiento del cauce principal generando una mayor superficie afectada al humedal, cuyos límites se manifiestan de manera difusa hacia ambas márgenes (Figura 2).

En los casos de los humedales de la zona rural, se identifica un curso principal de escasa expresión acompañado por una red de microdrenaje el cual pudo ser identificado a partir de la topografía de detalle realizada a partir del relevamiento fotogramétrico (Figura 3). El curso principal presenta un gradiente de 3,5 10-4 en el caso del Cajaravillas y de 1,2 10-3 en el Chubichaminí.

Tal como se observa en la Tabla 3 y Figura 2, el ancho medio de los BDF Del Gato y Carnaval (Figura 2a y b) es relativamente menor que la de los BDF Cajaravillas y Chubichaminí (Figura 2c y d).

En los BDF Carnaval y Del Gato, no se observa la red de microdrenaje probablemente como consecuencia de la profundización, dragado y

Tabla 3. Parámetros de las subcuencas de los humedales estudiados.

Table 3. Morphometric parameters of the riverine wetlands

\begin{tabular}{lcccc}
\hline & Carnaval & Del Gato Cajaravillas Chubichaminí \\
\hline Area Humedal $\left(\mathrm{m}^{2}\right)$ & 20015 & 81355 & 337 & 190.6 \\
Longitud del humedal $(\mathrm{m})$ & 843 & 1451 & 1277 & 1171 \\
Ancho medio $(\mathrm{m})$ & 82 & 95 & 128 & 158 \\
Diferencia de altura & 0,28 & 0,18 & 0,05 & 0,03 \\
Pendiente \% en el humedal & $3,010^{-2}$ & $1,210^{-2}$ & $3,510^{-4}$ & $1,210^{-3}$ \\
Profundidad del nivel freático & 15,45 & 18,65 & 0,45 & 0,36 \\
\hline
\end{tabular}



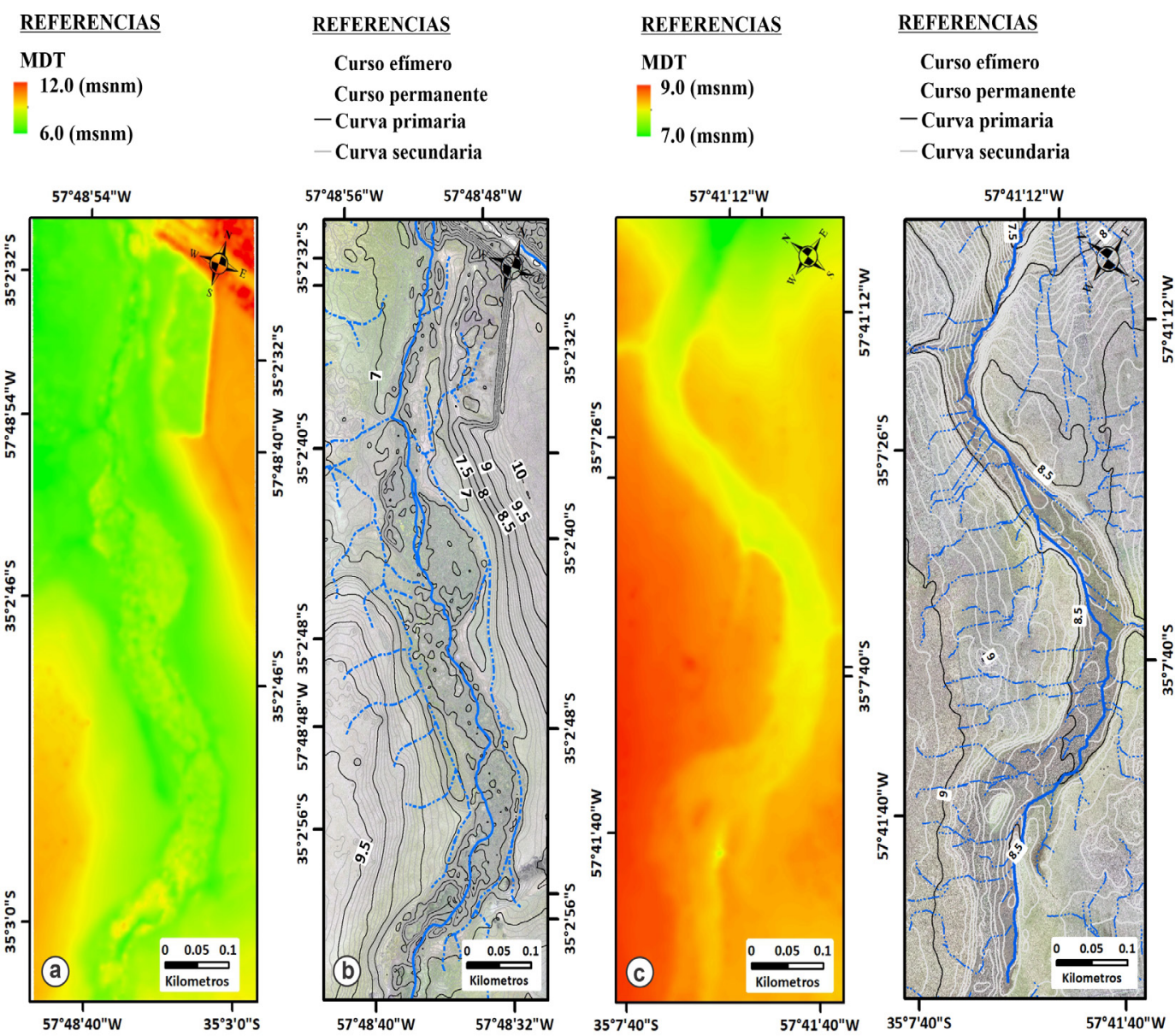

Figura 3. Modelo digital del terreno, mapa topográfico y red de drenaje de los arroyos. a y b Cajaravillas; c y d Chubichaminí.

Figure 3. Digital terrain model, topographic map and drainage network of the streams. a and $\mathbf{b}$ Cajaravillas; c and d Chubichaminí.

rectificación del curso principal (Figura 2a y b). Los resultados obtenidos a partir de los sondeos manuales realizados permitieron definir que el nivel freático se halla a una profundidad mayor a los $8 \mathrm{~m}$ b.n.t., lo cual es consistente con los mapeos regionales del agua subterránea. En este sentido, la falta de aporte subterráneo al caudal base condiciona la permanencia y desarrollo de estos humedales, los cuales solo reciben el aporte proveniente de la escorrentía superficial.

En el caso de los BDF Cajaravillas y Chubichaminí los sondeos manuales realizados permitieron identificar un nivel freático el cual se haya a escasos centímetros por debajo del terreno.
Con el objeto de verificar la relación entre el BDF y al agua subterránea, en la Figura 4 se muestran dos situaciones hidrológicas para el BDF del arroyo Chubichaminí, correspondientes al 14 de marzo y 2 junio de 2018 (Figuras 4a y b) y la evolución de los niveles freáticos para un sondeo de monitoreo ubicado próximo al curso de agua (F1). En el lapso existente entre los dos relevamientos se produjo una disminución en el espesor de la zona no saturada de aproximadamente 1,5 m. Asimismo, se pueden reconocer sectores con agua en superficie y sectores con diferente contenido de humedad. El detalle de las imágenes captadas por el dron permite diferenciar las zonas que 

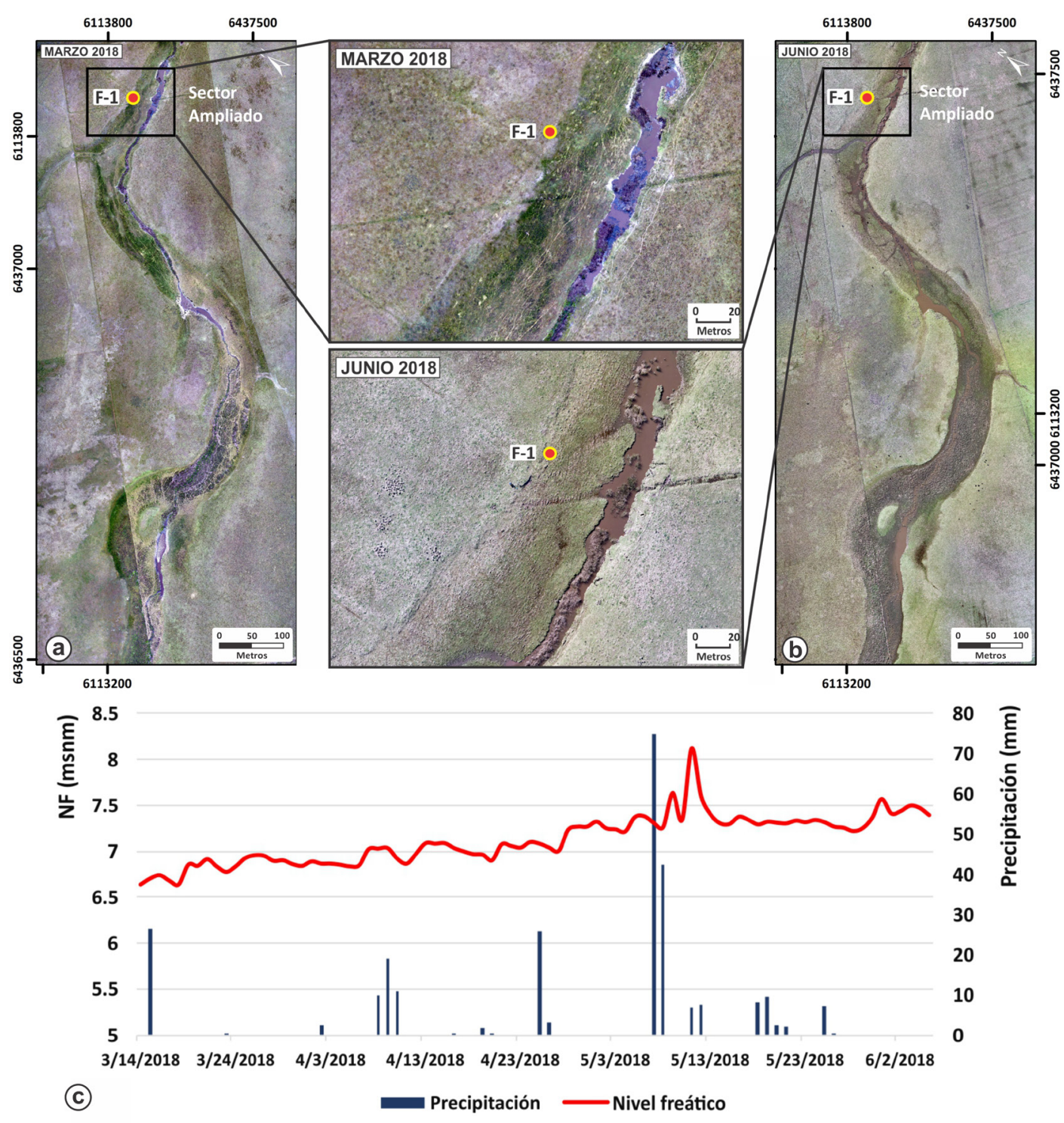

Figura 4. Ortomosaicos del sector de humedal del arroyo Chubichaminí para los meses a. marzo y b. junio de 2018. En c, se muestra la evolución del nivel freático registrado por el sensor automático instalado en la perforación de monitoreo F-1 (Circulo amarillo).

Figure 4. Orthomosaics of the Arroyo Chubichaminí wetland sector for the months, $\mathbf{a}$. March and $\mathbf{b}$. June of 2018. In c, the evolution of the water table recorded by the automatic sensor installed in the monitoring well F-1 (yellow circle) is shown.

presentan acumulación de agua en superficie y las variaciones observadas entre ambas fechas, las áreas del humedal que se relacionan con los cambios de la posición del nivel freático y las variaciones del tipo y estadio de la vegetación. Esta condición también se comprueba a partir de datos hidroquímicos que demuestran una similitud ente la composición del agua superficial y subterránea de los BDF (Figura 5). En ambos casos el carácter bicarbonatado sódico de las aguas subterráneas se corresponde con el de los humedales. Esta condición permite reconocer la dependencia de la componente subterránea por parte de estos humedales, de modo tal que cualquier intervención que se realice sobre el sistema acuífero impactará de manera directa 


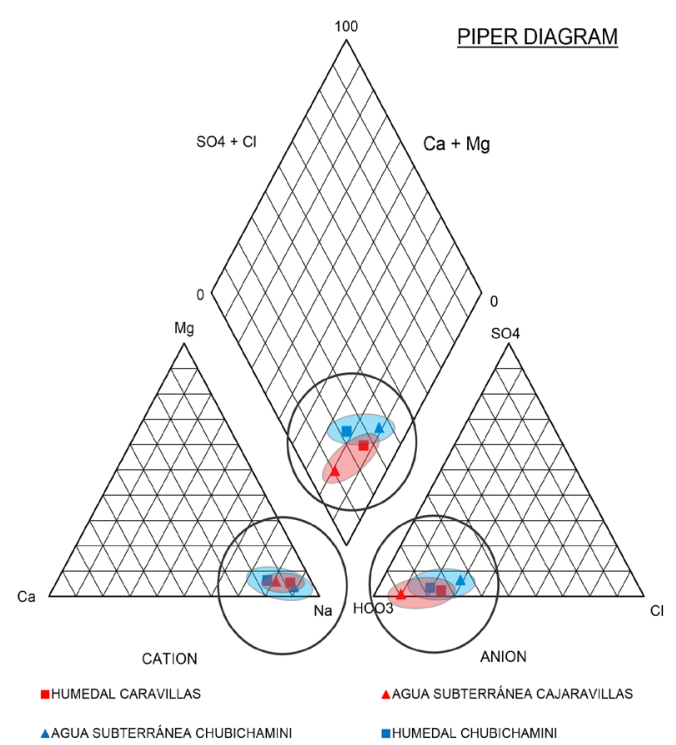

Figura 5. Diagrama de Piper. Muestreo de aguas subterráneas y superficiales realizados sobre los humedales de los arroyos Cajaravillas y Chubichaminí. Figure 5. Piper diagram. Sampling of groundwater and surface water carried out on the wetlands of the Cajaravillas and Chubichaminí streams.

sobre las condiciones de humedad en superficie, como así también en los ecosistemas en el que se desarrollan estos BDF.

Con el objeto de conceptualizar la relación agua superficial y subterránea en los BDF estudiados, en la Figura 6 se muestra un perfil esquemático para ambas situaciones hidrológicas. Se ejemplifica al BDF Chubichaminí como un humedal asociado al afloramiento del nivel freático, mientras que al BDF Carnaval como una condición sin una influencia directa del agua subterránea.

En el primer caso (Figura 6a), se observa que, de acuerdo con el balance hidrológico, y partiendo de una precipitación media anual de 1060 mm, la evapotranspiración alcanza los 783 mm, la infiltración es de 96,5 mm, mientras que la sumatoria del escurrimiento superficial y subterráneo es de 180,5 mm.

En el arroyo Chubichaminí (Figura 6b), tanto las precipitaciones, la evapotranspiración y la infiltración se mantienen similar respecto al anterior esquema. En este caso, el aporte de las aguas subterráneas $(96,5 \mathrm{~mm})$ al caudal base del arroyo genera un incremento en el caudal aguas abajo y por tanto una disminución del flujo subterráneo en la desembocadura. Esta condición, se pone de manifiesto a partir de verificación de un caudal superficial permanente el cual pudo ser advertido durante los trabajos de campo, incluso en diferentes épocas del año.

\section{Calidad del agua}

Las características fisicoquímicas del agua de los BDF demostraron que en el grupo Carnaval - del Gato las concentraciones de fósforo y nitrógeno son significativamente mayores, que se evidencia particularmente en las concentraciones de $\mathrm{PO}_{4}$ $y$ de $\mathrm{NH}_{4}{ }^{+}(p<0,001)$. En los BDF Chubichaminí - Cajaravillas se observaron valores más altos en la conductividad, porcentaje de saturación de oxígeno disuelto y DQO $(p<0,001)$ (Tabla S1, suplementaria). Por otra parte, estos últimos presentan concentraciones más elevadas de sólidos en suspensión (BDF Cajaravillas).

Durante el muestreo de marzo de 2018 los BDF Del Gato y Carnaval carecían de agua como consecuencia de la intensa sequía por la que atravesó el área de estudio razón por la cual no se cuenta con registros.

\section{DISCUSIÓN Y CONCLUSIONES}

Una caracterización y comprensión de los procesos geohidrológicos con una visión aplicada a las particularidades ecológicas de los bañados de desborde fluvial como los estudiados, requiere plantear metodologías que incluyan distintas escalas espaciales y temporales para su análisis. Por lo tanto, es necesario contemplar en una primera instancia un análisis global en un marco regional de la cuenca de drenaje para en sucesivas aproximaciones alcanzar un mayor detalle en los sitios específicos de estudio.

Las particularidades hidrodinámicas e hidroquímicas que se registran en los BDF son el resultado de procesos hidrológicos que ocurren en su entorno. La selección de BDF localizados en una zona rural y otros influenciados por la agricultura y la urbanización se empleó para reconocer los efectos de las alteraciones 
(a)
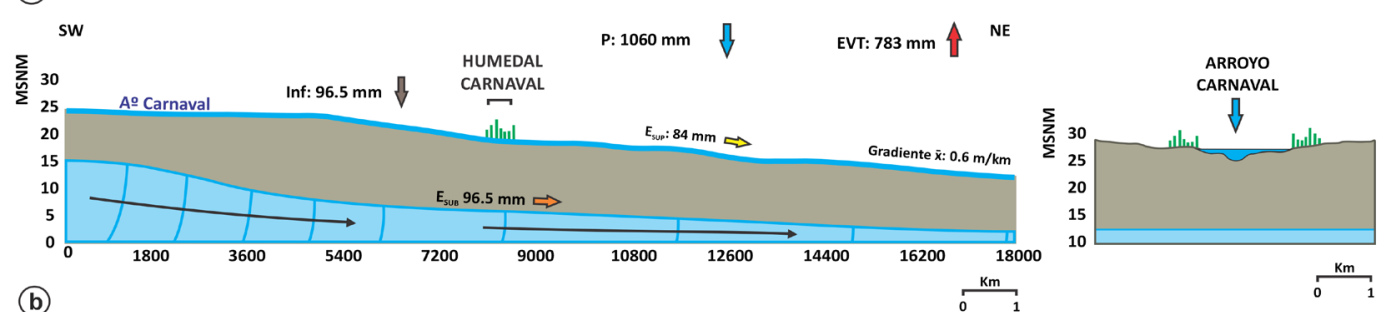

(b)
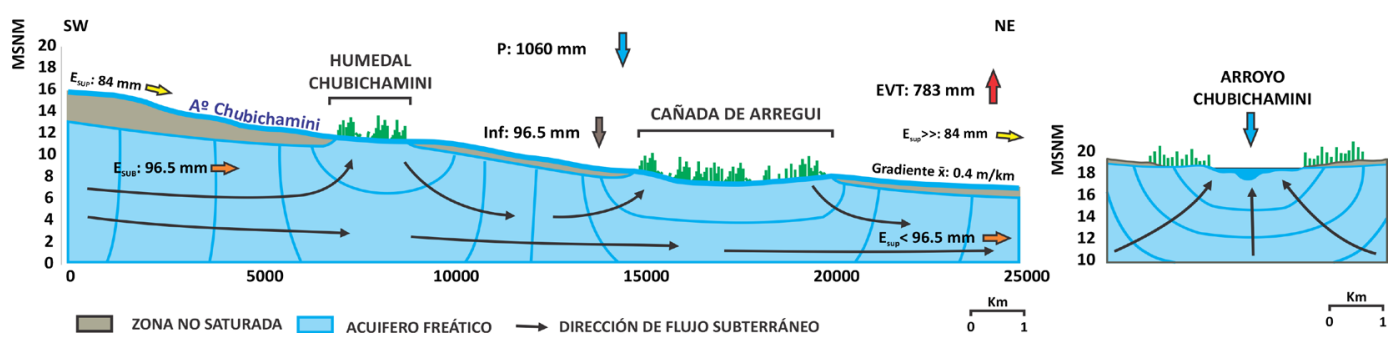

Figura 6. Modelo conceptual de funcionamiento hidrológico para las secciones longitudinales de los arroyos. a. Carnaval y b. Chubichaminí.

Figure 6. Conceptual model of hydrological functioning for longitudinal sections of streams. a. Carnival and $\mathbf{b}$. Chubichaminí.

hidrológicas generadas por las intervenciones humanas y sus consecuencias en el ecosistema asociado a dichos humedales. En tal sentido un análisis comparativo de las situaciones indicadas permitió definir las características geohidrológicas de una de ellas con condiciones próximas a las naturales y la otra fuertemente afectada por la actividad del hombre.

En el primer caso los BDF Cajaravillas y Chubichaminí muestran la existencia de agua en superficie en forma permanente, presentando una expansión en períodos húmedos y retracción en épocas secas. En estos humedales el nivel freático se sitúa a escasa profundidad o directamente está aflorante y sus fluctuaciones se asocian a las variaciones en las precipitaciones. La vinculación entre el sistema superficial y subterráneo se verifica también a partir de la similitud hidroquímica de las aguas subterráneas con respecto a las del humedal. En el área de aporte hacia estos BDF predomina la infiltración, produciéndose escurrimiento superficial sólo con lluvias esporádicas de alta intensidad en las que se supera la capacidad de infiltración del suelo. En estos casos los BDF actúan como cuerpos de almacenamiento que amortiguan los picos de las crecidas agua abajo. En los otros casos estudiados (BDF Carnaval y Del Gato) se destacan las alteraciones geohidrológicas asociadas a la modificación del uso de la tierra, vinculados a los cultivos hortícolas, canalizaciones, impermeabilización y la sobreexplotación del agua subterránea. En estos casos la presencia de agua en superficie es intermitente y se asocia a las precipitaciones 0 al vertido de aguas residuales. El nivel freático se encuentra profundo ( $>8 \mathrm{~m}$ ) y no influye en el estado de humedad del BDF ni tampoco en la calidad química del agua. La significativa explotación de agua subterránea origina la profundización de los niveles freáticos. Las características químicas del agua se asocian a las condiciones impuestas por los vertidos de aguas residuales y por los efectos derivados de las prácticas agrícolas (Ej: aumento en el P y N). La intensa actividad hortícola desarrollada en el entorno de estos humedales contribuye al ingreso de nutrientes que promueven la eutrofización del cuerpo de agua. Este tipo de prácticas también acarrean el empleo de fungicidas, herbicidas e insecticidas muchos de los cuales quedan retenidos en los BDF convirtiéndose en una amenaza para la calidad del agua y para la biota residente (Mac Loughlin et al., 2017; Rimoldi et al., 2018) Es reconocido en la bibliografía que el uso de la tierra agrícola degrada las corrientes al aumentar los aportes no puntuales de contaminantes, impactando 
en el hábitat del curso fluvial y su ribera. (Allan, 2004). Por otra parte, la impermeabilización del terreno y la disposición de cultivos bajo cubierta aceleran el escurrimiento superficial disminuyendo las posibilidades de infiltración para la recarga de agua subterránea. Asimismo, la construcción de canales de drenaje disminuye las posibilidades de regulación de las crecidas por parte de los BDF.

Se concluye que este comportamiento geohidrológico resulta un factor significativo en el deterioro de los ecosistemas. Por otra parte, los cursos afectados por alteraciones antrópicas (Ej: dragados, canalizaciones, etc.) han perdido su morfología natural y su conectividad con el estuario del Río de la Plata particularmente en su tramo inferior. Este efecto resulta relevante para la biota ya que se modifican las condiciones naturales, por ejemplo, existen especies de peces que emplean estos cursos como corredores biológicos (Paracampo et al., 2020). Si bien la mitigación de los efectos producidos sobre los ecosistemas ya degradados resulta una tarea compleja, es necesario un uso racional de los recursos a nivel de cuenca hidrográfica. La experiencia obtenida en los BDF con un fuerte deterioro de los ecosistemas lleva a la necesidad de comprender que el desarrollo socioeconómico sobre aquellos cuerpos que aún mantienen condiciones próximas a las naturales estén sujetos a la aplicación de pautas que derivan de una gestión integrada de cuencas y una planificación urbana sustentable. Los problemas de inundaciones, preservación de caudales y calidad del agua, incremento productivos y urbanización, deben resolverse a través de soluciones basadas en la naturaleza. En tal sentido se pueden incluir para ello entre otras prácticas, aquellas relacionadas con la creación de zonas de anegamientos para retener los episodios relacionados con lluvias de alta intensidad, prácticas de protección y estabilización de los cauces y la determinación de áreas de reservas para la explotación de aguas subterráneas.

Finalmente, la conservación de estos pequeños BDF vinculados a los arroyos requieren un diagnóstico y caracterización de las posibles alteraciones antrópicas y una comprensión de los problemas hídricos a escala regional donde el avance de la urbanización y la agricultura plantean permanente conflictos ambientales.

\section{BIBLIOGRAFÍA}

Allan, D. J. (2004). Landscapes and riverscales: the influence of land use on stream ecosystems. Annual Review of Ecological and Evolutionary Systems, 35, 257-284.

Bazán, J. M., Alberino, J. C., Varriano, N., Cariello, J, Kruse, E., Rojo, A., Deluchi, M., y Laurencena, P. (2011). Cuencas del arroyo El Pescado y Del Gato en los partidos de La Plata, Berisso y Ensenada. Tercera Reunión Anual PROIMCA - Primera Reunión Anual PRODECA. Mendoza.

Clesceri, L. S., Greenberg, A. E. \& Eaton, A. D. (Eds) (1998). Standard methods for the examination of water and wastewater. Washington: American A.D. Public Health Association (APHA).

Cochero, J., Di Giorgi1, H., Donadelli, J. H., Suárez, J., Simonetti, S., Finkler N. R. y Gasparini Fernandes Cunha, D. (2020). El rol de los bañados de desborde fluvial en la retención de nutrientes y su actividad metabólica. Biología Acuática, 35.

Fertonani, M. E. y Prendes, H. (1983). Hidrología en área de Ilanura. Aspectos conceptuales, teóricos y metodológicos. En: Fuschini Mejía, M. C. (Ed.). Hidrología de las Grandes Llanuras (pp. 787-864). Coloquio de Olavarría. UNESCO. Secretaría Nacional de Recursos Hídricos.

Gasparini Fernandes Cunha. D., Finkler, N. R., Gómez, N., Cochero, J., Donadelli, J. L., Saltarelli, W. A., Calijuri, M. C., Miwa, A. C. P., Tromboni, F., Dodds, W.K., Boëchat, I. G., Gücker, B., \& Thomas, S.A. (2019). Agriculture influences ammonium and soluble reactive phosphorus retention in South American headwater streams. Ecohydrology, 13(2). https:// doi.org/10.1002/eco.2184

Gómez, N., Rodrigues Capítulo, A., Colautti, D., Mariñelarena, A., Licursi, M., Cochero, J., Armendariz, L., Maroñas, M., Donadelli, J., 
Jensen, R., García De Souza, J., Maiztegui, T., García, I., Sathicq, M. B., Suarez, J. y Cortese, B. (2016). La puesta en valor de los servicios ecosistémicos que ofrecen los arroyos de llanura como una medida de mitigación de las inundaciones: el caso del $A^{\circ}$ del Gato en el Partido de La Plata. En: Volpedo, A., de Cabo, L; Arreghini, S. y Fernández Cirelli, A. (Eds). Ecología y manejo de ecosistemas acuáticos pampeanos (pp. 39 - 52). Ciudad Autónoma de Buenos Aires.

Kruse, E. y Laurencena, P. (2005). Aguas superficiales. Relación con el régimen subterráneo y fenómenos de anegamiento. En: Relatorio del XVI Congreso Geológico Argentino - Geología y Recursos Minerales de la Provincia de Buenos Aires. (pp. 313-326).

Kruse, E. y Zimmermann, E. (2002). Hidrogeología de Grandes Llanuras. Particularidades en la Llanura Pampeana (Argentina). In: Workshop publication on Groundwater and Human Development (pp. 2025-2038). Mar del Plata: XXXII IAH Congress.

Laterra, P., Booman, G. C., Picone, L, Videla, C., Orúe, M. E. (2018). Indicators of nutrient removal efficiency for riverine wetlands in agricultural landscapes of Argentine Pampas. Journal of Environmental Management, 222,148154.
Laurencena, P., Deluchi, M., Rojo, A. y Kruse, E. (2010). Influencia de la explotación de aguas subterráneas en el sector periurbano de La Plata. Revista de la Asociación Geológica Argentina, 66 (4), $484-489$.

Mac Loughlin, T. M., Peluso, L., Marino, D. J. (2017. Pesticide impact study in the peri-urban horticultural area of Gran La Plata, Argentina. Science of the Total Environment, 598, 572-580

Mackereth, F. J., Heron, F. J. H. \& Talling, J. F. (1978). Water analysis: some revised methods for limnologists. Ambleside: Freshwater Biological Association.

Paracampo, A., Marrochi, N., García, I., Maiztegui, T., Carriquiriborde, P., Bonetto, C. \& Mugni, H. (2020). Fish assemblages in Pampean streams (Buenos Aires, Argentina): Relationship to abiotic and anthropic variables. Annals of the Brazilian Academy of Sciences, 92(2), $1-17$.

Rimoldi, F., Peluso, L., Rossini, G. B., Ronco, A. E. \& Demetrio, P. M. (2018). Multidisciplinary approach to a study of water and bottom sediment quality of streams associated with mixed land uses: Case study Del Gato Stream, La Plata (Argentina). Ecological Indicators, 89,188-198. 